\title{
Study of Some Hyndantion Derivatives as Anticonvulsant Agents
}

\author{
Abida' ${ }^{1}$ Md Tauquir Alam ${ }^{1}$ and Mohammad Asif ${ }^{*}$ \\ 1 Department of Pharmaceutical Chemistry, Faculty of Pharmacy, Northern Border University, Rafha \\ 91911, PO Box 840, Saudi Arabia \\ ${ }^{2}$ Department of Pharmacy, Himalayan Institute of Pharmacy and Research, Dehradun, (Uttarakhand), \\ 248007, India
}

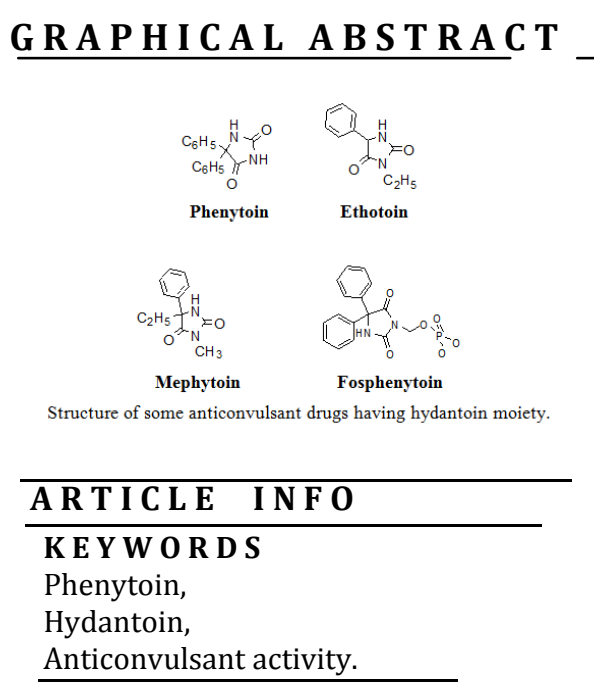

A B S T R A C T

Hydantoin derivatives are commonly used anticonvulsant drugs. It is generally effective against partial and tonic-clonic seizures but not in absent seizures. Phenytoin is the main drug of this group and other drugs Ethotoin, Mephenytoin also commonly used in the therapy of epilepsies. However, they have some adverse effects and long period of therapy to seizure control. To sort out these problems, various new hydantoin derivatives as well as prodrugs like Fosphenytoin were prepared. Phenytoin is effective in some cases of trigeminal and related neuralgias. Phenytoin is also used in the treatment of cardiac arrhythmias.

\section{Introduction}

Epilepsy is one of the most common neurological
disorders, affecting about $1 \%$ of the world's
population and characterized by recurrent seizure
attacks [1]. It is estimated that in one-third of these
patients, seizures are not adequately controlled by
existing drugs. Furthermore, the drugs available
have shown significant side effects, and many have
narrow therapeutic indices and are difficult to
formulate. To exert its anti-convulsant effect, the
drug must reach its receptors in the central
nervous system (CNS). Yet, many of the drugs
exhibit physicochemical and protein-binding
properties that would not permit crossing of the
blood-brain barrier (BBB) [2]. Phenytoin is one of
the most widely used drugs in the therapy of
epilepsy. However, its low solubility in water, both
as free acid and sodium salt, makes its administration difficult to patients and it has been seldom satisfactory. Phenytoin is given orally as sodium salt in a strong alkaline solution, since it requires a $\mathrm{pH}$ between 10 and 12 to maintain in solution. The alkalinity of this dosage form often causes gastric irritation, which is a serious drawback. Phenytoin can also be given by the intramuscular route, but the product commonly precipitates at the injection site, leading to unreliable blood levels of the drug. Moreover, absorption of intramuscular phenytoin is very slow, it is not appropriate for treating epileptic seizures, in which a loading dose of the product is required. For parenteral use, sodium phenytoin is formulated in aqueous alkaline solution ( $\mathrm{pH} 12)$ containing $40 \%$ of propylene glycol and $10 \%$ of ethanol. The risks associated with the use of this formulation are

\footnotetext{
* Corresponding author: Mohammad Asif

Tel number: +91-9897088910, E-mail: aasif321@gmail.com 
obvious, taking into account its high $\mathrm{pH}$ as well as the precipitation of the free acid [3].The classic prodrug approach to improve membrane permeability of drug molecules employs lipophilic derivatives to increase passive membrane penetration. In recent years, different nutrient transporters (i.e. oligopeptide, amino acid, and glucose transporters) have been identified and cloned. The active nutrient transport systems have become a target for prodrug design [4]. The feasibility of utilizing natural amino acids and benzhydrols as a promoiety refers to the fact that can transport a model compound across the BBB.

\section{Hydantoin drugs:}

The actual chemotherapy of epilepsy dates back to 1850 s with the introduction of 'inorganic bromides'. It is, however, remarkable here that the therapeutic gainful application of 'phenobarbital' around 1920s virtually made an era, making meaningful treatment of epilepsy. Almost within a span of two decades the wonderful contributions made by Merritt and Putman were recognized when they discovered that the '5-substituted hydantoins' were capable of suppressing the electrically induced convulsions in the laboratory animals. This ultimately paved the way towards the synthesis of 5, 5-diphenylhydantoin (or phenytoin) which possessed the best as well as least sedative activity.

Phenytoin: Phenytoin (diphenylhydantoin, Dilantin) is effective against all types of partial and tonic-clonic seizures but not absence seizures. It was found to exert its action on the motor cortex where it stabilizes the neuronal membrane and thereby inhibits the spread of the seizure discharge. Present evidence also suggests that it limits high frequency repetitive firing by blocking $\mathrm{Na+-}$ channels in a used and frequency dependent fashion. Besides, it enhances Ca- binding to the phospholipids present in neuronal membranes. In fact, these effects collectively give rise to a more stable membrane configuration. Importantly, these critical findings are found to be in perfect harmony with the glaring and supportive fact that it is most easily demonstrated characteristic features are by virtue of its ability to limit the development of maximal seizure activity and to minimize the virtual extension of the seizure phenomenon from the active focus. Interestingly, both of these splendid features in phenytoin are very much related to the clinical usefulness beyond any reasonable doubt [5-12].

\section{History}

Phenytoin was first synthesized in 1908 by Biltz, but its anticonvulsant activity was not discovered until 1938. In contrast to the earlier accidental discovery of the antiseizure properties of bromide and phenobarbital, phenytoin was the product of a search among non-sedative structural relatives of phenobarbital for agents capable of suppressing electroshock convulsions in laboratory animals. It was introduced for the treatment of epilepsy in the same year. Since this agent is not a sedative in ordinary doses, it is maintained that antiseizure drugs need not induce drowsiness and encourage the search for drugs with selective antiseizure action.

Structure-Activity Relationship: A 5-phenyl or other aromatic substituent appears essential for activity against generalized tonic-clonic seizures. Alkyl substituents in position 5 contribute to sedation, a property absent in phenytoin. The carbon 5 position permits asymmetry, but there appears to be little difference in activity between isomers.

\section{Pharmacological Effects}

Central Nervous System. Phenytoin exerts antiseizure activity without causing general depression of the CNS. In toxic doses, it may produce excitatory signs and at lethal levels a type of decerebrate rigidity. The most significant effect of phenytoin is its ability to modify the pattern of maximal electroshock seizures. The characteristic tonic phase can be abolished completely, but the residual clonic seizure may be exaggerated and prolonged. This seizure-modifying action is observed with many other antiseizure drugs that are effective against generalized tonic-clonic seizures. By contrast, phenytoin does not inhibit clonic seizures evoked by pentylenetetrazol. 


\section{Mechanism of Action}

Phenytoin limits the repetitive firing of action potentials evoked by a sustained depolarization of mouse spinal cord neurons maintained in vitro[13]. This effect is mediated by reducing the rate of recovery of voltage-activated $\mathrm{Na}^{+}$channels from inactivation, an action that is both voltage- (greater effect if membrane is depolarized) and usedependent. These effects of phenytoin are evident at concentrations in the range of therapeutic drug levels in cerebrospinal fluid (CSF) in humans, which correlate with the free (or unbound) concentration of phenytoin in the serum. At these concentrations, the effects on $\mathrm{Na}^{+}$channels are selective, and no changes of spontaneous activity or responses to iontophoretically applied GABA or glutamate is detected. At concentrations five- to tenfold higher, multiple effects of phenytoin are evident, including reduction of spontaneous activity and enhancement of responses to GABA; these effects may underlie some of the unwanted toxicity associated with high levels of phenytoin.

\section{Pharmacokinetic Properties}

Phenytoin is available in two types of oral formulations that differ in their pharmacokinetics: rapid-release and extended-release forms. Oncedaily dosing is possible only with the extendedrelease formulations, and due to differences in dissolution and other formulation-dependent factors, the plasma phenytoin level may change when converting from one formulation to another. Confusion also can arise because different formulations can include either phenytoin or phenytoin sodium. Therefore, comparable doses can be approximated by considering "phenytoin equivalents," but serum level monitoring is also necessary to assure therapeutic safety.

The pharmacokinetic characteristics of phenytoin are influenced markedly by its binding to serum proteins, by the nonlinearity of its elimination kinetics, and by its metabolism by CYPs. Phenytoin is extensively bound (about 90\%) to serum proteins, mainly albumin. Small variations in the percentage of phenytoin that is bound dramatically affect the absolute amount of free (active) drug; increased proportions of free drug are evident in the neonate, in patients with hypoalbuminemia, and in uremic patients. Some agents, such as valproate, can compete with phenytoin for binding sites on plasma proteins; when combined with valproate-mediated inhibition of phenytoin metabolism, marked increases in free phenytoin can be the result. Measurement of free rather than total phenytoin permits direct assessment of this potential problem in patient management. Phenytoin is one of the few drugs for which the rate of elimination varies as a function of its concentration (i.e., the rate is nonlinear). The plasma half-life of phenytoin ranges between 6 and 24 hours at plasma concentrations below $10 \mu \mathrm{g} / \mathrm{ml}$ but increases with higher concentrations; as a result, plasma drug concentration increases disproportionately as dosage is increased, even with small adjustments for levels near the therapeutic range. The majority (95\%) of phenytoin is metabolized principally in the hepatic endoplasmic reticulum by CYP2C9/10 and to a lesser extent CYP2C19. The principal metabolite, a parahydroxy phenyl derivative, is inactive. Because its metabolism is saturable, other drugs that e metabolized by these enzymes can inhibit the metabolism of phenytoin and increase its plasma concentration. Conversely, the degradation rate of other drugs that are substrates for these enzymes can be inhibited by phenytoin; one such drug is warfarin, and addition of phenytoin to a patient receiving warfarin can lead to bleeding disorders. An alternative mechanism of drug interactions arises from phenytoin's ability to induce diverse CYPs; coadministration of phenytoin and medications metabolized by these enzymes can lead to an increased degradation of such medications. Of particular note in this regard are oral contraceptives, which are metabolized by CYP3A4; treatment with phenytoin could enhance the metabolism of oral contraceptives and lead to unplanned pregnancy. The potential teratogenic effects of phenytoin underscore the importance of attention to this interaction. Carbamazepine, oxcarbazepine, phenobarbital, and primidone also can induce CYP3A4 and likewise might increase degradation of oral contraceptives.

The low aqueous solubility of phenytoin hindered its intravenous use and led to production of fosphenytoin, a water-soluble prodrug. 
Fosphenytoin(cerebyx) is converted into phenytoin by phosphatases in liver and red blood cells with a half-life of 8 to 15 minutes. Fosphenytoin is extensively bound (95\% to $99 \%$ ) to human plasma proteins, primarily albumin. This binding is saturable and fosphenytoin displaces phenytoin from protein binding sites. Fosphenytoin is useful for adults with partial or generalized seizures when intravenous or intramuscular administration is indicated.

Toxicity. The toxic effects of phenytoin depend on the route of administration, the duration of exposure, and the dosage. When fosphenytoin, the water-soluble prodrug, is administered intravenously at an excessive rate in the emergency treatment of status epilepticus, the most notable toxic signs are cardiac arrhythmias, with or without hypotension, and/or CNS depression. Although cardiac toxicity occurs more frequently in older patients and in those with known cardiac disease, it also can develop in young, healthy patients. These complications can be minimized by administering fosphenytoin at a rate of less than $150 \mathrm{mg}$ of phenytoin sodium equivalents per minute, a rate that therefore should not be exceeded. Acute oral overdosage results primarily in signs referable to the cerebellum and vestibular system; high doses have been associated with marked cerebellar atrophy. Toxic effects associated with chronic treatment also are primarily dose-related cerebellar-vestibular effects but also include other CNS effects, behavioral changes, increased frequency of seizures, gastrointestinal symptoms, gingival hyperplasia, osteomalacia, and megaloblastic anemia. Hirsutism is an annoying untoward effect in young females. Usually, these phenomena can be diminished by proper adjustment of dosage. Serious adverse effects, including those on the skin, bone marrow, and liver, probably are manifestations of drug allergy. Although rare, they necessitate withdrawal of the drug. Moderate elevation of the plasma concentrations of hepatic transaminases sometimes are observed; since these changes are transient and may result in part from induced synthesis of the enzymes, they do not necessitate withdrawal of the drug.
Gingival hyperplasia occurs in about $20 \%$ of all patients during chronic therapy and is probably the most common manifestation of phenytoin toxicity in children and young adolescents. It may be more frequent in those individuals who also develop coarsened facial features. The overgrowth of tissue appears to involve altered collagen metabolism. Toothless portions of the gums are not affected. The condition does not necessarily require withdrawal of medication and can be minimized by good oral hygiene. A variety of endocrine effects have been reported. Inhibition of release of antidiuretic hormone (ADH) has been observed in patients with inappropriate ADH secretion. Hyperglycemia and glycosuria appear to be due to inhibition of insulin secretion. Osteomalacia, with hypocalcemia and elevated alkaline phosphatase activity, has been attributed to both altered metabolism of vitamin $\mathrm{D}$ and the attendant inhibition of intestinal absorption of $\mathrm{Ca}^{2+}$. Phenytoin also increases the metabolism of vitamin $\mathrm{K}$ and reduces the concentration of vitamin $\mathrm{K}$ dependent proteins that are important for normal $\mathrm{Ca}^{2+}$ metabolism in bone. This may explain why the osteomalacia is not always ameliorated by the administration of vitamin D. Hypersensitivity reactions include morbilliform rash in $2 \%$ to $5 \%$ of patients and occasionally more serious skin reactions, including Stevens-Johnson syndrome. Systemic lupus erythematosus and potentially fatal hepatic necrosis have been seldom reported. Hematological reactions include neutropenia and leukopenia. A few instances of red-cell aplasia, agranulocytosis, and mild thrombocytopenia have been reported. Lymphadenopathy, resembling Hodgkin's disease and malignant lymphoma, is associated with reduced immunoglobulin A (IgA) production. Hypoprothrombinemia and hemorrhage have occurred in the newborns of mothers who received phenytoin during pregnancy; vitamin $\mathrm{K}$ is effective treatment or prophylaxis.

\section{Plasma Drug Concentrations}

A good correlation usually is observed between the total concentration of phenytoin in plasma and its clinical effect. Thus, control of seizures generally is obtained with concentrations above $10 \mu \mathrm{g} / \mathrm{ml}$, 
while toxic effects such as nystagmus develop at concentrations around $20 \mu \mathrm{g} / \mathrm{ml}$.

Drug Interactions. Concurrent administration of any drug metabolized by CYP2C9 or CYP2C10 can increase the plasma concentration of phenytoin by decreasing its rate of metabolism. Carbamazepine, which may enhance the metabolism of phenytoin, causes a well-documented decrease in phenytoin concentration. Conversely, phenytoin reduces the concentration of carbamazepine. Interaction between phenytoin and phenobarbital is variable.

Therapeutic Uses: Phenytoin is one of the more widely used antiseizure agents, and it is effective against partial and tonic-clonic but not absence seizures. The use of phenytoin and other agents in the therapy of epilepsies is discussed further at the end of this chapter. Phenytoin preparations differ significantly in bioavailability and rate of absorption. Some cases of trigeminal and related neuralgias appear to respond to phenytoin, but carbamazepine may be preferred in using phenytoin in the treatment of cardiac arrhythmias.

Ethotoin: It is N-dealkylated and parahydroxylatedin vivo. However, the $\mathrm{N}$-dealkyl metabolite is most presumably the 'active compound'; it is similarly metabolized by parahydroxylation, and the resulting hydroxyl function undergoes conjugation subsequently. This particular 'drug substance' is used against not-sospecific seizures, but invariably on an adjunctive basis because of its low potency. In a broader perspective, such anticonvulsants, which are not completely branched on the appropriate $\mathrm{C}$-atom, are of definite lower potency than their rather more fully branched structural analogues.

Mephenytoin: It is metabolically $\mathrm{N}$-dealkylated to the corresponding 5-ethyl-5-phenylhydantoin, which is considered the 'active agent'. Interestingly, the metabolized product, happens to be the 'hydantoin counterpart' of phenobarbital as one of the first breed of hydantoins ever introduced into the therapeutic armamentarium. Furthermore, it may be assumed that 'mephenytoin' is a 'pro-drug' that essentially ameliorates a part of its toxicity along with skin and blood disorders of serious nature of the delivered 'active drug'. The metabolic inactivation of this drug and its corresponding dimethylmetabolite is caused due to the parahydroxylation and subsequent conjugation of the free hydroxyl moiety.

Fosphenytoin(Cerebyx) is a prodrug that is highly soluble in intravenous solutions without solubilizing agents and is supplied in vials for intravenous use. Fosphenytoin is converted to phenytoin following parenteral administration. It is very effective in terminating seizures and will stop most status epilepticus episodes and provide longterm control without any decreased level of consciousness. All of these drugs should be administered slowly to avoid respiratory depression and apnea.

\section{Various anticonvulsant drugs contain hydantoin derivatives:}

Hybrids between phenytoin and thiosemicarbazide, 1,3,4-oxadiazole, 1,3,4thiadiazole or 1,2,4-triazole were tested for anticonvulsant activity. Some compounds showed the highest protection (80\%) in the subcutaneous pentylenetetrazole (scPTZ) test at a dose of 100 $\mathrm{mg} / \mathrm{kg}$, whereas few drugs displayed promising anticonvulsant effect in the maximal electroshock (MES) model [14]. Some derivatives of phenytoin were synthesized by the alkylation of phenytoin with substituted methylene bromides. These compounds were found as anticonvulsant active in the MES and scPTZ induced seizures [15].The synthesis of phenytoin-3-carboxylic acid (1) derivatives with methyl esters of alanine (2), phenylalanine (3), and tyrosine (4) and with benzhydrol (5) and chlorbenzhydrol (6) was carried out. Some phenytoin derivatives, methyl 2(2,5-dioxo-4,4-diphenyl imidazolidine-1carboxamido) propanoate (2), methyl 2-(2,5-dioxo4,4-diphenyl-imidazolidine-1-carboxamido)-3phenylpropanoate (3), methyl-2-(2,5-dioxo-4,4diphenyl-imidazolidine-1-carboxamido)-3-(4hydroxyphenyl) propanoate (4) and benzhydryl2,5-dioxo-4,4-diphenyl-imidazolidine-1carboxylate(5) and4-chlorobenzhydryl- 2,5-dioxo4,4-diphenyl-imidazolidine-1-carboxylate These phenytoin derivatives were exhibited 
anticonvulsant activity. The pharmacological screening of the phenytoin derivatives (2),(3),(4),(5) and(6) indicated that the amino acids (especially phenylalanine and alanine) and benzhydrol linked with phenytoin are actually increasing the anticonvulsant activity of phenytoin and decreasing the neurotoxicity. The linkage with chlorobenzhydrol and tyrosine was found ineffective in modifying the anticonvulsant activity of phenytoin positively [16]. However, the efficacy is lowered by the erratic bioavailability after oral administration. Cyanoguanidino-phenytoin (CNGDPH) was prepared by the replacement of the urea moiety by a cyanoguanidine moiety and evaluated the bioavailability and anticonvulsant activity. CNGDPH is poorly water-soluble and has a pKa of 5.3 at $\mathrm{pH}$ 7.4. The Pharmacokinetic parameters were obtained after oral administration of CNG-DPH to rats and were compared to those of phenytoin after administration of an equimolar amount. AUC, $t_{\max }$, and $\mathrm{C}_{\max }$ significantly increased compared to those of phenytoin. The anticonvulsant profile was similar to the profile of phenytoin. CNG-DPH was active in the MES test, albeit 7-fold less active than phenytoin. The analogue did not protect animals against convulsions induced by chemicals such as PTZ, picrotoxin, N-methyl-aspartate, strychnine, and bicuculline. The bioisosteric exchange of the urea moiety of the molecule with the cyanoguanidine moiety dramatically changed the physicochemical and pharmacokinetic parameters compared to those of phenytoin, the concomitant change of the affinity toward molecular targets reduced the pharmacological activity and the therapeutic efficacy of the compound [17]. The anticonvulsant activity of phenytoin-lipid conjugates obtained by covalent binding of 3hydroxy-methylphenytoin to dimyristoylglycerides via a succinidyl linkage, to 2-(1,3dimyristoylglyceryl)butyric acid and to 3myristoyl-2-myristoylmethylpropionic acid was evaluated in the MES test and ScPTZ. The phenytoin-lipid conjugates were less active than the parent drug in the MES test after i.p administration as suspensions, but exhibited comparable activity when injected as a solution in dimethylsulphoxide. They also protected mice from
MES-induced seizures following oral administration of aqueous suspensions of the compounds. The anticonvulsant activity could be correlated to in-vitro pancreatic lipase-mediated hydrolysis. The bis-deacyl derivatives were at least as active but, in some cases, even more toxic than phenytoin. Oral administration of two of the lipid conjugates resulted in a faster onset of the anticonvulsant activity compared with the administration of an equimolar dose of phenytoin itself. All compounds were inactive in the scPTZ test. It is concluded that the lipids act as prodrugs of phenytoin, which is generated by lipolysis upon oral administration [18].

A series of bicyclic derivatives based on 5,5diphenylhydantoin (DPH) and/or 5-arylidenehydantoin skeletons (BZH) were exhibited potential anticonvulsant activities. In preliminary pharmacological tests a few of these agents showed some anticonvulsant activities, like the parent DPH. The electronic parameters (molecular electrostatic potential, MEP, and dipole moment orientation) for the DPH molecule were used as a model differed significantly from those calculated for the bicyclic molecules [19]. The pharmacokinetic characteristics of phenytoin (DPH) and its derivatives, DPH-1-methylnicotininate (MNDPH), valeroyl DPH (VADPH) and valproyl DPH (VPDPH) have protein binding characteristics. The hydrolytic conversion of these derivatives to DPH was also studied using small intestine, liver and brain tissues, as well as rat plasma. The log partition coefficient (PC) values of all derivatives were much higher than DPH. The most fractions of VADPH and VPDPH existed as an ionized form at physiological $\mathrm{pH}$, whereas MNDPH existed as a unionized form under the same conditions. The binding data of DPH and its derivatives to both rat plasma protein and bovine serum albumin (BSA) exhibited straight lines over their concentration ranges used, indicating that DPH and its derivatives have a single binding site on the protein. The binding potencies of the derivatives to both proteins were much greater than DPH. No DPH produced from VADPH and VPDPH was found in the biological fluids over a period of $24 \mathrm{~h}$. 
<smiles>CCCCCC1(P)NC(=O)NC1=O</smiles>

Phenytoin

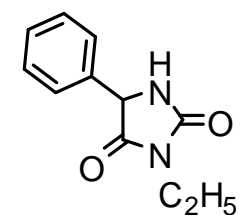

Ethotoin

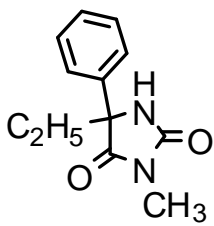

mephytoin

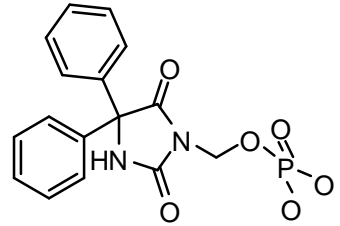

Fosphenytoin

Fig.1.Structure of some anticonvulsant drugs having hydantoin moiety.

However, the hydrolysis of MNDPH to DPH was observed in plasma and the tissues used, with the most rapid hydrolysis in the small intestine and the hydrolysis rate constant in plasma was ca. 20 -fold greater than that in the brain [20].Phenytoin-lipid conjugates obtained by covalent binding of hydroxymethylphenytoin to diacylglycerides and to 3-acyloxy-2-acyl-oxymethylpropionic acids formed. In contrast to the corresponding bis-deacyl derivatives, the lipids were not significantly hydrolyzed in aqueous buffers and in plasma. Incubation with pancreatic lipase yielded primarily the bis-deacyl compounds, which are comparable to monoglycerides, and subsequently liberated phenytoin. The glyceride-derived prodrugs were better substrates for the enzyme compared with the 3-acyloxy-2-acyloxymethyl-propionic acid derivatives. It is concluded that the phenytoin lipid conjugates are hydrolyzed by pancreatic lipase in a similar manner as natural triglycerides [21]. Iminohydantoins selectively substituted at position C-5 and their 1-carbobenzoxy derivatives have been evaluated for their anticonvulsant activity in mice. Lipophilic 1-carbobenzoxy iminohydantoins were more potent than the unsubstituted counterparts were. The individual enantiomers of the chiral iminohydantoins showed that the anticonvulsant activity resided primarily in the $S$ isomers. The (S)-(+)-1-carbobenzoxy-5-isobutyl-2iminohydantoin was the most active member. This compound was not nearly as active as phenytoin against MES induced convulsions, but was also active against PTZ-induced seizures, a broader clinical potential. The closest analogue of phenytoin, viz., 5,5-diphenyl-2-iminohydantoin, failed to show any significant activity. Methylation on $\mathrm{N}-3$ or the imino nitrogen of 5,5-diphenyl-2iminohydantoin also did not provide a compound with substantial activity. 2-Thiophenytoin was not active against MES seizures and showed only a weak activity against PTZ. The SAR of 2- iminohydantoins was quite different from that of 2hydantoins [22]. A well-known side effect of the long-term therapy with phenytoin is gum hyperplasia. 1-Acyl compounds and esters of the phydroxymetabolite with aliphatic and aromatic carboxylic acids can be potential prodrugs for therapeutic use if the neoformation of connective tissue is intended [23].

The in-vivo metabolic conversion of equal mixture of phenytoin and decadeuteriophenytoin to the para-hydroxy metabolite in rat was investigated in order to verify a possible role of an insertion or abstraction mechanisms in the hydroxylation process. This gives evidence of the arene oxide intermediacy possibly being the sole pathway for para-hydroxylation of phenytoin [24].Some 5,5-diarylhydantoins and their 3-amino derivatives have improved rotational restriction of the benzene moieties. These compounds symmetrically substituted in the benzene rings proved practically inactive as anticonvulsants. High and prolonged activity was observed with 3 -amino5,5-diphenylhydantoin (7)[25].Anticonvulsant activities of 3-methylphenytoin (8) (3-MP) and 1,3dimethylphenytoin(9) (1,3-DMP) were observed after i.p. administration of the drugs dissolved in dimethylsulphoxide (DMSO), while maximal activity was obtained within $15 \mathrm{~min}$ with phenytoin. The time course of increase in plasma phenytoin levels correlated with that of anticonvulsant activities. It was also found that 1,3DMP gave rise to a major unidentified metabolite as well as 3-MP and phenytoin. Mice injected with high doses of 3-MP $(100 \mathrm{mg} / \mathrm{kg})$ in DMSO exhibited severe epileptiform activities. This shows that 3-MP is at least a pro-convulsant, taking into account that its effects might have been enhanced by DMSO. Unlike phenytoin, 3-MP lacked the ability to inhibit synaptosomal uptakes of both glutamate and GABA. This difference may be related to the fact that 
phenytoin, but not 3-MP, possesses potent anticonvulsant activity [26].The problems related to phenytoin metabolites at the influence of the $p$ hydroxyphenyl metabolite of phenytoin (HPPH) on the metabolic disposition of the parent compound, and the relationship of the dihydrodiol metabolite to the putative presence of arene oxides [27].The 1benzenesulfonyl-5,5-diphenylhydantoin

having a potent anti-inflammatory activity was compared with that of Phenytoin, an antiepileptic drug. Compound $\mathbf{1 0}$ showed a potent anticonvulsant activity against MES seizure, the activity being comparable to that for Phenytoin [28].Phenytoin derivatives containing at the position 3 aminoalkyl, aminohydroxyalkyl and amide groups, these compounds, given in a dose of $50 \mathrm{mg} / \mathrm{kg}$ were not protected against PTZ seizures [29]. Alkoxymethyl, acyloxymethyl, and mixed alkylalkoxymethyl or alkylacyloxymethyl derivatives of 5-ethyl-5-phenylhydantoin exhibit anticonvulsant activity and proved effective are bis (alkoxymethyl) and mixed alkylalkoxy methyl derivatives of 5,5-diphenylhydantoin. The 1,3bis(methoxymethyl)-5,5-diphenyl-hydantoin (11) and 3-acetoxymethyl-5-ethyl-5-phenyl hydantoin(12) showed good activity against MES seizures, and 3-methoxymethyl-5-ethyl-5phenylhydantoin is effective against both MES and PTZ. No compounds showed greater activity against MES seizures than the parent compounds [30].The3-aryl-5-benzylidene-2-thiohydantoins

(13) were prepared by condensation of 3-o-tolyland 3-m-tołyl-2--thiohydantoin with aromatic aldehydes. Derivatives of 2-thiohydantoin showed weak anticonvulsant activity in PTZ test and shown no protecting activity against the MES [31].Six ringfluorinated phenytoinanalogs, and their anticonvulsant activity in the MES and sc PTZ seizure assays, 5-(4-Fluorophenyl)-5phenylhydantoin (14), 5-(3-fluorophenyl)-5phenylhydantoin (15), and 5,5-bis(4-fluorophenyl) hydantoin(16) were active in the MES assay. The compounds were less active than phenytoin but showed very long duration of action [32].The N3alkyl- or arylpiperazinoalkyl substituted derivatives of 5,5-diphenylhydantoin weakly affect the motility and hypermotility induced by amphetamine. They did not show the anticonvulsive activity [33].
The water-soluble phenytoin derivatives, bearing ionizable groups were showed the hydrolysis of amino esters is very fast than fosphenytoin [34]. Fosphenytoin is a phosphate ester prodrug of phenytoin developed as a replacement for standard injectable sodium phenytoin. After absorption, phenytoin is cleaved (conversion half-life 8-15 min) from fosphenytoin. Unlike phenytoin, fosphenytoin is freely soluble in aqueous solutions and rapidly absorbed by the i.m route. Fosphenytoin has been tested successfully for three indications in humans: i.m maintenance dosing, i.m loading dose administration, and i.v treatment of status epilepticus. Local toxicity (pain, burning, itching) is less by the i.m or i.v route for fosphenytoin than for injectable sodium phenytoin. Systemic toxicity is similar to both preparations except that hypotension is less common and paresthesias are more common with fosphenytoin [35]. Fosphenytoin was designed to overcome many of the shortcomings associated with parenteral phenytoin sodium. Specifically, fosphenytoin is a highly water-soluble, phosphate ester of phenytoin that has no known pharmacologic activity before its conversion to phenytoin. Dosing of fosphenytoin uses phenytoin equivalents (PE) to minimize dosage errors when converting from the conventional formulation. It is rapidly and completely converted to phenytoin after i.v and i.m dosing, half-lives after i.v administration range from 8-15 minutes. The absorption rate appears to be the rate-limiting step in the conversion of fosphenytoin to phenytoin after i.m administration (half-life range 22-41 min).

Bioavailability of phenytoin derived from both i.v and i.mfosphenytoin is essentially $100 \%$. As a consequence of concentration-dependent protein binding, fosphenytoin is bioequivalent to phenytoin sodium at i.v infusion rates of 100-150 mg $\mathrm{PE} /$ minute and $50 \mathrm{mg} /$ minute, respectively. The fosphenytoin is safe and significantly better tolerated than phenytoin sodium when administered i.v. It is also well tolerated when given i.m, and this is a valuable alternative route of administration when i.v is accessible. 


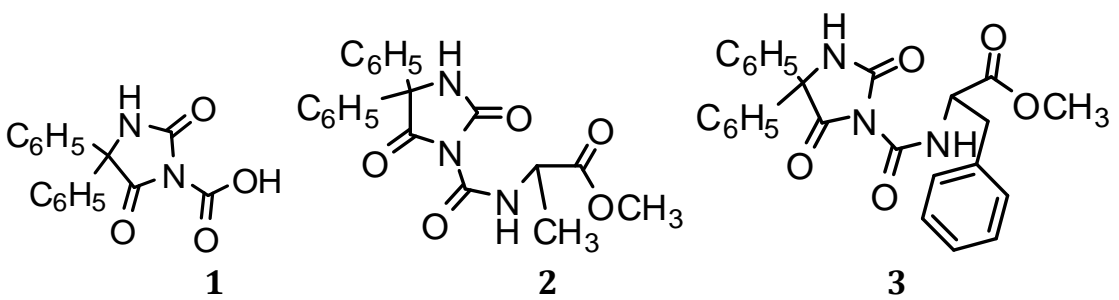<smiles>COC(=O)C(Cc1ccc(O)cc1)NC(=O)N1C(=O)NC(S)(S)C1=O</smiles>

4<smiles>NN1C(=O)NC(c2ccccc2)(c2ccccc2)C1=O</smiles>

7<smiles>CN1C(=O)NC(c2ccccc2)(c2ccccc2)C1=O</smiles>

8

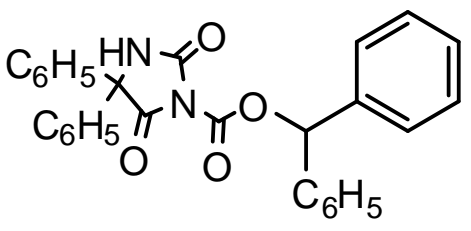

5<smiles>CN1C(=O)N(C)C(c2ccccc2)(c2ccccc2)C1=O</smiles>

9

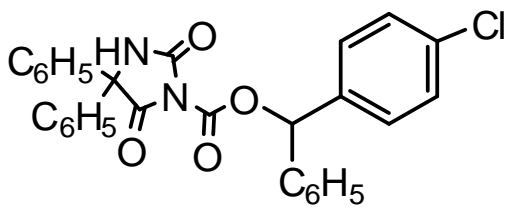

6<smiles>O=C1NC(=O)C(c2ccccc2)(c2ccccc2)N1S(=O)(=O)c1ccccc1</smiles>

10<smiles>COCN1C(=O)N(COC)C(c2ccccc2)(c2ccccc2)C1=O</smiles>

11<smiles>CCC1(c2ccccc2)NC(=O)N(COC(C)=O)C1=O</smiles>

12

13<smiles>O=C1NC(=O)C(c2ccccc2)(c2ccc(F)cc2)N1</smiles>

14<smiles></smiles>

15
16

Fig. 2.Structure of some hydantoin derivatives with anticonvulsant activity.

Hence, fosphenytoin has the potential as a safe, well-tolerated, and effective means of delivering phenytoin parenterally in a variety of clinical settings [36].The protein binding and pharmacokinetics of diazepam, ACC-9653 (a phenytoin prodrug), and phenytoin were evaluated.
No significant differences were observed in the fraction unbound or pharmacokinetic parameters of ACC-9653, phenytoin, or diazepam when ACC9653 was administered alone compared to concomitant administration with diazepam. The phenytoin fraction unbound increased significantly 
with increased concentrations of ACC-9653, indicating displacement of phenytoin from its binding sites by ACC-9653. ACC-9653 also demonstrated concentration dependent binding. The lack of a significant pharmacokinetic drug interaction between ACC-9653 and diazepam suggests that these drugs may be safely administered together, although this conclusion should be confirmed in the intended patient population [37].

A phenytoin prodrug, 3-phosphoryloxymethyl phenytoin (ACC-9653; 1), has been developed with more favourable physicochemical properties than phenytoin for parenteral administration. Each patient received a single iv dose of undiluted 1 equivalent to their twice daily phenytoin dose (100-200 mg). An equivalent dose of i.m. 1 was administered in the gluteus maximums muscle one week later. Serial blood samples were obtained after each dose [40]. ACC-9653, the disodium phosphate ester of 3-hydroxymethyl-5,5diphenylhydantoin, is a prodrug of phenytoin with advantageous physicochemical properties. ACC9653 is rapidly converted enzymatically to phenytoin in vivo. ACC-9653 and phenytoin sodium have equivalent anticonvulsant activity against seizures induced by MES in mice following i.p., oral, or i.v. administration. The ED50 doses were 16 $\mathrm{mg} / \mathrm{kg}$ for i.v. ACC-9653 and $8 \mathrm{mg} / \mathrm{kg}$ for i.v. phenytoin sodium. ACC-9653 and phenytoin sodium have similar antiarrhythmic activity against ouabain-induced ventricular tachycardia in anesthetized dogs. Only phenytoin sodium displayed in vitro antiarrhythmic activity against strophanthidin-induced arrhythmias in guinea pig right atria. In anesthetized dogs, a high dose of ACC$9653(31 \mathrm{mg} / \mathrm{kg})$ was infused over 15,20 , and 30 min and the responses were compared to an equimolar dose of phenytoin sodium $(21 \mathrm{mg} / \mathrm{kg})$. The ACC-9653 and phenytoin sodium treatments produced similar marked reductions in diastolic blood pressure and contractile force. The maximum effects of each treatment occurred at the time of maximum phenytoin sodium levels. Acute toxicity studies of ACC-9653 and phenytoin sodium were carried out in mice, rats, rabbits, and dogs by i.v., i.m., and i.p. routes of administration. The systemic toxic signs of both agents were similar and occurred at approximately equivalent doses. Importantly, the local irritation of ACC-9653 was markedly less than phenytoin sodium following i.m. administration [41].The bioavailability of phenytoin from ACC-9653 versus i.v administered sodium phenytoin was determined for i.v and intramuscular administration of ACC-9653 to healthy volunteers. Absolute bioavailability of phenytoin derived from ACC-9653 in each subject was calculated as the ratio of the phenytoin area under the plasma concentration time curve for time 0 to infinity [AUC(0-inf)] after ACC-9653 divided by the phenytoin AUC(0-inf) after i.v sodium phenytoin. The mean absolute bioavailability of ACC-9653 was 0.992 after i.v administration and 1.012 after i.m administration. The bioavailability of ACC-9653 is complete following i.v or i.m administration in single-dose volunteer studies. The absolute bioavailability of phenytoin derived from ACC-9653 in subjects with therapeutic plasma phenytoin concentrations is being studied in patients given simultaneous infusions of stable isotope-labelled tracer doses of ACC-0653 and sodium phenytoin [42].The phenytoin (PHT) solution has many disadvantages stemming from poor aqueous solubility of PHT. A novel approach to solve the problem has been the synthesis of a phosphate ester of PHT (PHT prodrug ACC-9653). This water-soluble compound is metabolized rapidly into PO4 and PHT. Patients received an i.v. or i.m. dose of ACC-9653 at a dose equivalent to the patients' morning dose of PHT. After a period of 6 days, during which patients were again maintained with oral PHT, they were given a dose of ACC-9653 via whichever route they had not yet received. The $\mathrm{T}_{\max }$ of the prodrug averaged 5.7 and $36 \mathrm{~min}(0.095$ and $0.606 \mathrm{~h}$ ) after i.v. and i.m. administrations, respectively. The elimination half-life of ACC-9653 (conversion from prodrug to PHT) after i.v. and i.m. administration was 8.4 and $32.7 \mathrm{~min}(0.140$ and $0.545 \mathrm{~h}$ ), respectively, and both were independent of the dose [43]. A prodrug of phenytoin, the disodium phosphate ester of 3-hydroxymethyl-5,5diphenylhydantoin (ACC-9653) and phenytoin were measured in plasma samples. The concentration of phenytoin reached $90 \%$ of its maximum about 12 minutes after the end of the infusion of ACC-9653. At the dose of $1200 \mathrm{mg}$ of prodrug, the average peak concentration of 
phenytoin was about $17 \mu \mathrm{g} / \mathrm{mL}$, near the upper limit of the therapeutic range. Adverse reactions, lightheadedness, nystagmus, incoordination were minor and attributed to phenytoin [44].

\section{DISCUSSION}

The chemistry of nitrogen heteroatom containing aromatic compounds is becoming more popular in the area of research. Hydantoin derivatives and related compounds have shown good anticonvulsant or antiepileptic activities mainly against grand mal epilepsy and some other activities. They bind to physiological targets or receptors, producing many possible mechanisms of actions. Hydantoins are inexpensive and easily synthesized, and therefore have been examined as anticonvulsant activities. A slight variation in the substitution pattern on the hydantoin nucleus often causes a difference in activities and therefore hydantoin with various substituents are being synthesized and tested for activities in search of better medicinal agents and great interest has arisen in the design and synthesis of new hydantoin compounds to explore their potent activities against epilepsy $[45,46]$. The hydantoin nucleus, which has a useful structure for further molecular

\section{REFERENCRES}

[1]. B. Malawska, Curr Topics Med Chem, 5(2005), 69.

[2]. E. Shek, Adv. Drug Delivery Rev, 14(1994), 227.

[3]. J. Bosch, Bioorg. Med. Chem. Lett., 9(1999), 1859.

[4]. C. Yang, and A. Mitra, J. Pharm. Sci., 90 (2001), 340.

[5]. R.V. Wagh, R.V. Antre, R.J. Oswal, and H.M. Nimje, J Pharm Res \& Bio-Sci Res, 1(3): 2011, 142-147.

[6]. A.C. Gerlach, and J.L. Krajewski Pharmaceuticals, 3(2010), 2884-2899.

[7]. M. Bialer, Epilepsy Res, 15(2006):637-647.

[8]. D. Chisholm, Epilepsia, 46 (2005), 751-759.

[9]. M.A. Rogawski, and W. Loscher, Nat. Rev. Neurosci., 5(2004), 553-564.

[10]. P. Kwan, and J.W. Sander, J. Neurol. Neurosurg. Psychiatry, 75(2004), 1376-1381.

[11]. C. Dwivedi Am. J. Pharm. Educ., 65(2001), $197-$ 202.

[12]. R.L. Macdonald, and L.J. Jr. Greenfield, Curr. Opin. Neurol., 10 (1997), 121-128. exploration for the development of new derivatives with anticonvulsant activities, has received much attention [5-9]. In order to further explore chemical space available for pharmaceutical applications, there is a continued demand for the development of new hydantoins that have novel structures to medicinal chemistry development.

\section{CONCLUSION}

The hydantoinnucleus, due to its easy fictionalization at various position of ring has found considerable pharmaceutical interest as the core structure of antiepileptic compounds. The ring system now became interesting in search of new and more potent compounds with lesser side effect. The chemists, pharmacologists and researchers have attention to hydantoin compounds due to their antiepileptic potential. The ptherapeutical profile has attracted the attention of many researchers to explore this nucleus. It can be concluded that hydantoin has a great potential.

\section{Conflict of interests}

The authors declare that there is no conflict of interests regarding the publication of this paper

[13]. M.J. McLean, and R.L. Macdonald, J. Pharmacol. Exp. Ther., 238(1986), 727-738.

[14]. S. Botros, N.A. Khalil, B.H. Naguib, Y. El-Dash, Eur J Med Chem., 60 (2013), 57-63.

[15]. C.0. Usifoh, Arch Pharm (Weinheim), 334(2001):366-8.

[16]. M. Deodhar, P. Sable, A. Bhosale, K. Juvale, R. Dumbare, P. Sakpal, Turk J Chem, 33(2009), 1-7.

[17]. D.M. Lambert, B. Masereel, B. Gallez, M, Geurts, and G.K. Scriba, J Pharm Sci., 85(1996), 1077-81.

[18]. G.K. Scriba, D.M. Lambert, and J.H. Poupaert, J Pharm Pharmacol, 47(1995), 197-203.

[19]. J, Karolak-Wojciechowska, W. Kwiatkowski, and K. Kiec-Kononowicz, Pharmazie, 50(1995), 1147.

[20]. T. Ogiso, T. Tanino, M. Iwaki, O. Muraoka, and G. Tanabe, Biol Pharm Bull., 17(1994), 1425-9.

[21]. G.K. Scriba, Pharm Res., 10(1993):1181-6.

[22]. C.H. Kwon, M.T. Iqbal, and J.N. Wurpel. J Med Chem., 34(1991), 1845-9. 
[23]. W. Fürst, and R. Wildner. Pharmazie, 46(1991), 424-6.

[24]. M.A. Moustafa, A.A. el-Emam, H.I. Subbagh, and M.K. el-Din, Arzneimittelforschung, 40(1990), 1076-8.

[25]. J. Lange, and J. Lapszewicz, Acta Pol Pharm., 47(1990), 31-4.

[26]. P.T. Wong,, S.T. Tan, and H.S. Lee. Jpn J Pharmacol., 48(1988), 473-8.

[27]. A.J. Glazko, Ther Drug Monit., ;9(1987), 407-15.

[28]. H. Fujioka, T. Tan, M. Kishi, H. Miyazaki, Y. Masuda, and Y. Yokoyama. J Pharmacobiodyn, 9(1986), 303-14.

[29]. K. Kieć-Kononowicz, E. Stypuła, J. Krupińska, and B. Cebo, Pol J Pharmacol Pharm. 1985, 37(1985)693-9.

[30]. R. Jalilian, M. Shahmari, A. Taheri, K. Gholami, Ultrason Sonochem, 61(2020) 104802.

[31]. R. Jalilian, A. Taheri, e-Polymers, 18(2018) $123 .$.

[32]. W.L. Nelson, Y.G. Kwon, G.L. Marshall, J.L. Hoover, and G.T. Pfeffer. J Pharm Sci, 68(1979), 115-7.

[33]. A. Zejc, M. and Pawlłowski, Pol J Pharmacol Pharm, 32(1980),173-8.

[34]. J. Bosch, T. Roca, J. Domènech, and M. Suriol. Bioorg Med Chem Lett,. 5(1999), 1859-62.

[35]. T.R. Browne. Clin Neuropharmacol., 20(1997), 112.

[36]. B.A. Boucher, Pharmacother, 16(1996), 777-91.
[37]. E.K. Hussey, G.E. Dukes, J.A. Messenheimer, K.L. Brouwer, K.H. Donn, T.F. Krol, and L.J. Hak, Pharm Res, 7(1990), 1172-6.

[38]. B.D. Jamerson, K.H. Donn, G.E. Dukes, J.A. Messenheimer, K.L. Brouwer, and J.R. Powell, Epilepsia, 31(1990),592-7.

[39]. N.Y. Walton, and D.M. Treiman, Epilepsy Res, 5(1990), 165-8.

[40]. B.A. Boucher, A.M. Bombassaro, S.N. Rasmussen, C.B. Watridge, R. Achari, and P. Turlapaty, J Pharm Sci., 78(1989),929-32.

[41]. S.S.H. Davarani, Z. Rezayati-zad, A. Taheri, N. Rahmatian, Materials science \& engineering C, Materials for biological applications, 71(2017) 572-83.

[42]. T.R. Browne, H. Davoudi, K.H. Donn, C.L. Dougherty, G.E. Dukes, B. Evans, J.E. Evans, B. Jamerson, J. Kres, C.M. McEntegart, et al., Epilepsia. 30 Suppl2(1989), S27-32.

[43]. I.E. Leppik, R. Boucher, B.J. Wilder, V.S. Murthy, C.A. Rask, C. Watridge, N.M. Graves, R.J. Rangel, and P. Turlapaty, Epilepsia. 30Suppl2 (1989), S22-6.

[44]. N. Gerber, D.C. Mays, K.H. Donn, A. Laddu, R.M. Guthrie, P. Turlapaty, C.Y. Quon, and W.K. Rivenburg, J ClinPharmacol.,28(1988) 1023-32.

[45]. S. Rump, I. Ilczuk, K. Walczyna, E. Zadrozyńska, Monogr Neural Sci., 5(1980),163-7.

[46]. K. Kieć-Kononowicz, and A. Zejc, Acta Pol Pharm., 38(1981), 49-53.

HOW TO CITE THIS ARTICLE

Abida, Md Tauquir Alam and Mohammad Asif, Study of Some Hyndantion Derivatives as Antivonvulsant Agents, Prog. Chem. Biochem. Res. 2020, 3(2), 93104.

DOI: $10.33945 /$ SAMI/PCBR.2020.2.2

URL: http://www.pcbiochemres.com/article 106475.html 